\title{
ORGANIZATIONAL CITIZENSHIP BEHAVIOR AND QUALITY OF WORK LIFE AMONG NURSES IN PORT SAID HOSPITALS
}

Noura Fawzy Elsayed Soliman El-Nahas ${ }^{1}$,Fatma Ahmed Abed Ali Osman ${ }^{2}$,

\author{
Sonia Mohamed Elsayed Elsayad ${ }^{3}$
}

B.Sc. Nursing, Port Said University, ${ }^{1}$, Professor of Nursing Administration

Faculty of Nursing -Cairo university ${ }^{2}$, Assist. Prof. of Psychiatric Nursing and Mental

Health -Faculty of Nursing- Port Said University

\begin{abstract}
Background: It is vital to increase nurses' quality of work life because this has the potential both to improve organizational citizenship and ensure an adequate nursing work force. Aim: To explore the relationship between organizational citizenship behavior and quality of work life among nurses in Port Said hospitals. Subjects and Method: A descriptive comparative correlation research design was utilized. Setting: This study was conducted in General Port Said hospital and Atta Specialized hospital. Subjects: A sample of 207 nurses who at job in time of the study. Tools of data collection: Two tools included organizational citizenship behavior scale and quality of work life components. Results: Showed that, high percentage of studied nurses agreed on organizational citizenship behavior scale. It was determined that the nurses obtained the highest score from the altruism, conscientiousness dimension followed by courtesy. Regarding quality of work life components, it was found that around half and more of the studied person agreed on overall quality of work life. Conclusion:There was positive correlation among organizational citizenship behavior and dimensions of quality of work life. Recommendation: Increase training programs in hospitals help nurses to achieve the required skills and improve communication among nurses.
\end{abstract}

Key Words: Organizational citizenship behavior, nurses and quality of work life. 


\section{INTRODUCTION}

Organizational Citizenship Behavior (OCB) is critical for the success of today's organization upon extant research has clearly revealed that, as it inherently strives from its employees to extend their discretionary behaviors in the form of Organizational Commitment (OC)beyond the expected normal duties. However, this kind of anticipated behavior of employees is somehow silently contradicting the notion of work-life balance (Pradhan, Jena, \&Kumari, 2016).

Also, organizational citizenship behavior can be of influence on the nurses' attitude and behaviors and guide their activities towards the hospital objectives accomplishment and eventually it can influence the quality of the services offered to the patients and creates good and conducive working environment that called Quality of Work Life (QWL) (Tabrsa, Esma'eeliGivi\&Esma'eeliGivi, 2010; Allameh, Sha'baniNaftchali, Khaza'ee Paul, VericKazemi, Amani, 2014).

Quality of working life is important managerial approaches that work towards alignment of employees with his organization, uphold the culture of excellence to improve performance, motivates the employees to learn further and helps in human resource development. Quality of Work Life (QWL) is a socio-psychological determinant of the quality of work and is defined as "the quality of relationship between the employees and the total working environment". It concerned with the overall climate of work and its impact on people as well as on organization effectiveness (Quadri, 2019).

According to Chowdhury, (2019) the purpose of QWL is to increase employee satisfaction, improve the physical and psychological health of employees who create positive feelings, improve employee productivity, strengthen learning in the workplace, Improve constantly management of change and transition, building the organization's image as the best in employee recruitment and retention and motivation.

Quality of work life creates conducive working environment to learn, grow, live and enjoy by fullest utilization of human potentiality that propel employees to exhibit individual as well as voluntary behavior in the form of employee commitment and loyalty known as organization citizenship behavior. These behaviors motivate 
employees to show over duties and contextual performance beyond the call of the duty (Abubakar, Anasori, \&Lasisi, 2019).

Moreover, According to Elamin, \&Tlaiss, (2015).QWL can be described as the impact of human and organizational effectiveness combined with an emphasis on participation in problem solving and decision-making as well as the performance evaluation of the human assets demands the employees to exhibit voluntary behavior, which is popularly referred as Extra Role Behavior (ERB) or is otherwise known as Organizational Citizenship Behavior (OCB).

\section{Significance of study:}

Organizational Citizenship Behavior amongst the most important factors that can direct behaviors, attitudes and interactions of nurses toward high quality services, Sois considered to be a reflection of the employees' commitment to their organization and encourage teamwork, promotes organizational communication, develops organizational environment, and reduces nurses errors (Dargahi, \&Shaham et al, 2012).On the other hand, the quality of work life is a comprehensive plan which enhances the satisfaction and learning of the staffs in the work environments. It also helps them in terms of the change (Davoudi, 2014).

As previously mentioned literature, it can be assumed that there is a scarcity in studies concerning organizational citizenship behavior and quality of work life among nurses in Egypt, which may contribute to attaining organizational goals and encourage employees for more efficient and motivated quality of work. Quality of Work Life (QWL) is essential for healthcare organizations. With high QWL organizations can achieve better productivity, become highly competitive and can seek ways to address the issues of recruitment and retention(Amini, \&Mortazavi, 2013).

During the study in the selected hospitals observed that nurses have demonstrably withdrawn their enthusiasm for productive work, and have committed themselves to a critical minimum effort. So, it is hoped that the findings would create an awareness of some of the possible explanation of the resignation from the job whilst nurses still continue to work,in addition to explore the relationship between organizational citizenship behavior and quality of work life among nurses in Port Said hospitals. 


\section{AIM OF THE STUDY:}

The study aims to explore the relationship between organizational citizenship behavior and quality of work life among nurses in Port Said hospitals.

\section{Objective of the study:}

Find out the relationship between organizational citizenship behavior and quality of work life among nurses in Port Said hospitals.

\section{SUBJECTS AND METHOD:}

A descriptive comparative correlation research design was used

\section{Study settings:}

This study was conducted at two different sectors of hospitals in Port Saidcity (public and private hospitals) namely:

1- Port Said General hospital: It consists of 21 departments, 18 of them are in-patient units and the other three departments are: the quality assurance unit, infection control unit and training unit.

2- Atta Specialized hospital: It consists of 4 departments, two of them are in-patient units, one emergency and one is operating department. There were chosen for availability and accessibility of the nurses.

\section{Study subjects:}

Convenient sample of nurses who are at job in the time of the study in Port Said general hospital and Atta specialized hospital with a total number of (207) nurses.

\section{TOOL OF DATA COLLECTION:}

Two main tools were used in the study for data collection. 


\section{Tool I: Organizational citizenship behavior scale:}

The internationally developed and validated questionnaire by(Podsakoff, 1990) and adapted by(Lee\& Allen, 2002) as well as translated to Arabic language by(Hamed, 2014).It consists of two parts as follows:

- The First Part: A Self structured interview questionnaire: This part aimed at collecting socio-demographic characteristics of nurses such as age, gender, marital status, working unit and years of experiences.

- The Second Part: Organizational citizenship behavior scale: This part aimed at assessing organizational citizenship behavior among nurses. The participant`s response to each sub item is on a five points Likert's scale ranging from (Strongly Disagree=1) to (Strongly Agree=5). It consists of thirty six items measuring five dimensions of organizational citizenship behaviors such as Altruism (1-7), Conscientiousness (8-15), Sportsmanship (16-19), Courtesy (20-26) and Civic virtue (loyalty) (27-36).

\section{Scoring system:}

Every statement was given a score; strongly agree was scored (5), disagree was scored (4) Neutral was scored (3)Agree was scored (2)and strongly disagree was scored (1). Ranges of response from agree to strongly agree. Total nurses response score was conducted according to total nurses response on the sheet. If the nurses total score from agree to strongly agree was considered response organizational citizenship behavior. If the nurses total score at the level of organizational citizenship behavior so their stratification will be judged as uncertain. If the total nurses scored strongly disagree or less was considered.

\section{Tool II: Quality of work life questionnaire:}

It was developed by Swamy, Swamy and Rashmi, (2015) and was translated into Arabic by the researcher. This tool was used to assess quality of work life among nurses. The participant's response to each sub item will be on a three points Likert's scale ranging from $($ Agree $=1)$ to $($ Disagree $=3)$

It consists of 50 statements measuring nine components of quality of work life as work environment (1-6), organization culture (7-13), relation and cooperation (14-19), training and development (20-24), compensation and rewards (25-29), facilities (30-34), 
job satisfaction and job security (35-41), autonomy of work (42-47) and adequacy of resources $(48-50)$.

\section{Scoring system:}

Every statement was given a score; Disagree was scored (3), Neutral was scored (2) Agree was scored (1). The scores of the items were summed up and the total divided by the number of the items giving a mean score for the part. These scores were converted into percent score. The domain was considered to be high score indicates a high degree score was if the percent is $75 \%$ or more, moderate degree score was if the percent $60 \%$ or lower than $75 \%$ and a lower degree if less than $60 \%$.

\section{Tool validity:}

It was ascertained by a jury consisting of five experts in the Administration Nursing field for clarity, relevance, applicability, comprehensiveness, understanding and ease for implementation, according to their suggestions, the modifications were applied.

\section{Reliability of Tools:}

Cronbach's alpha coefficient was calculated to assess the reliability of the developed tools through its internal consistency. The tools were proved to be reliable as the reliability of organizational citizenship behavior scale was 5.943 , and the reliability ofquality of work life questionnaire was 0.609

\section{Pilot study:}

Pilot study was carried out on 10.0\% (13 from Port Said general hospital and 7 from Atta hospital) of the total sample. The purposes of the pilot study were to test the applicability and clarify the feasibility of the study tools and it served to estimate the time needed to complete the tools. It also helped to find out any obstacles and problems that might interfere with data collection, based on findings of the pilot study, certain modification of the tools were done. Subjects included in the pilot study were excluded from the study subjects. Following this pilot study, the process of data collection was performed. Modifications of some words in the tools were done according to the results of the pilot study. 


\section{Field work:}

Data have been collected for 6 months throughout the period from of September2018 to the end of February2019. The data was collected during the 2 days per week. One day was permitted for each hospital to collect data needed from 10 AM to $1 \mathrm{PM}$.

\section{The field work has been performed in the following sequence:}

- Permission was taken from each hospital to carry out the study, the researcher met the medical director and nurse executive to explain the aim of the study and gain their support and cooperation.

- Then the nurses were met individually and invited to participate and obtained their consent to be recruited in the study after explaining the nature of the study, confidentiality and anonymity of their responses were ensured

- The researcher distributed the data collection forms with instruction about how to fill it.

- The data were collected one day per week during the morning shifts. The day was Thursday and work was done between 10:00 am to 1:00 pm. The researcher was available all the time to clarify any ambiguities.

\section{Ethical Considerations:}

Permission was taken from each hospital director to carry out the study. Also the aim of the study has been clarified to the headmasters to gain their support and cooperation. Purpose of the study was explained to each nurse and oral consent was obtained. Those who agreed to participate were assured about confidentiality and privacy, and they have the right to withdraw from the study at any time without giving any reasons. This information was used for research purpose only.

\section{Statistical analysis:}

Data were fed to the computer and analyzed using IBM SPSS software package version 20.0. (Armonk, NY: IBM Corp) Qualitative data were described using number and percent. Quantitative data were described using range (minimum and maximum), mean, and standard deviation. Significance of the obtained results was judged at the 5\% level. 


\section{RESULTS:}

Table (1): Illustrates the distribution of the studied nurses according to socio demographic data, it was shown that about half of the studied sample aged 30 - 39 years old. Age's mean \pm S.D was $33.12 \pm 7.43$. Most of them were married females, about half of them studied technical diploma 3 years; inpatient department, more than half of them had 15 years' experience and more. On the other side, socio-demographic characteristics at Atta specialized hospital, it was shown that above half of the studied sample aged 20 -29 years old. Age's mean \pm S.D was $27.47 \pm 5.16$. Above half of them were married females, about half of them studied bachelor of nursing; inpatient department, more than half of them had $>5$ years' experience.

Table(2): Showed that mean scoreof organizational citizenship behavior scale and socio demographic data in general Port Said hospitalthere were a statistically significant relations to department, but there were no statistically significant relations to age (years), gender, educational qualification, marital status and years of experiences. While at Atta specialized hospitalthere were a statistically significant relations to department, but there were no statistically significant relations to age (years), gender, educational qualification, marital status and years of experiences.

Table(3): Clarified that mean score of quality of work life components and socio demographic data in general Port Said hospitalthere were no statistically significant relations to age (years), gender, educational qualification, marital status and years of experiences. The only statistically significant relation was found to department. While at Atta specialized hospitalthere was no statistically significant relations to age (years), gender, educational qualification, department and marital status. The only statistically significant relation was found to years of experiences.

Table (4): Illustrates the comparison between the studied hospitals according to organizational citizenship behavior scale. Regarding altruism, conscientiousness, sportsmanship, courtesy, civic virtue and overall organizational citizenship behavior scale there were strongly agreed in general Port Said hospital with Atta specialized hospital.

Table (5): Shows that the comparison between the studied hospitals according to quality of work life components: Regarding organization culture, relation and 
cooperation and training and development, job satisfaction and job security, adequacy of resources and overall quality of work life components in general Port said hospital there were more agreed with Atta specialized hospital, while work environment, autonomy of work, compensation and rewards and facilities at Atta specialized hospital there were more agreed with general port said hospital.

Table (6): The table show that clear overall organizational citizenship behavior scale was statistically significant correlated to the overall quality of work life components.

Table (1): Distribution of the studied nurses according to socio demographic data in general Port Said hospital $(\mathrm{n}=137)$

\begin{tabular}{|c|c|c|}
\hline Socio demographic data & No. & $\%$ \\
\hline 1-Age (years) & & \\
\hline $20-29$ & 43 & 31.4 \\
\hline $30+-39$ & 64 & 46.7 \\
\hline $40-49$ & 29 & 21.2 \\
\hline $50+$ & 1 & 0.7 \\
\hline $\begin{array}{l}\text { Min. - Max. } \\
\text { Mean } \pm \text { SD. }\end{array}$ & \multicolumn{2}{|c|}{$\begin{array}{c}21.0-50.0 \\
33.12 \pm 7.43\end{array}$} \\
\hline 2-Gender & & \\
\hline Male & 6 & 4.4 \\
\hline Female & 131 & 95.6 \\
\hline \multicolumn{3}{|l|}{ 3-Maritalstatus } \\
\hline Single & 11 & 8.0 \\
\hline Married & 123 & 89.8 \\
\hline Divorced & 2 & 1.5 \\
\hline Widow & 1 & 0.7 \\
\hline \multicolumn{3}{|l|}{ 4-EducationalOualification } \\
\hline Technical Diploma 3 years & 81 & 59.1 \\
\hline Technical Diploma 5 years & 11 & 8.0 \\
\hline Nursing Technician Institute & 24 & 17.5 \\
\hline Institute of technical healthy & 5 & 3.6 \\
\hline Bachelor of nursing & 16 & 11.7 \\
\hline \multicolumn{3}{|l|}{ 5-Department } \\
\hline Neonatal & 13 & 9.5 \\
\hline Emergency & 22 & 16.1 \\
\hline I.C.U & 41 & 29.9 \\
\hline Inpatient & 61 & 44.5 \\
\hline Operation & 0 & 0.0 \\
\hline \multicolumn{3}{|l|}{ 6-Years of experiences } \\
\hline$>5$ & 29 & 21.2 \\
\hline $5-<10$ & 16 & 11.7 \\
\hline $10-<15$ & 24 & 17.5 \\
\hline$\geq 15$ & 68 & 49.6 \\
\hline
\end{tabular}


Table(1): Distribution of the studied nurses according to socio demographic data at Atta specialized hospital $(\mathrm{n}=70)$ "continue"

\begin{tabular}{|c|c|c|}
\hline Socio demographic data & No. & $\%$ \\
\hline 1-Age (years) & & \\
\hline $20-29$ & 54 & 77.1 \\
\hline $30+-39$ & 12 & 17.1 \\
\hline $40-49$ & 4 & 5.7 \\
\hline $50+$ & 0 & 0.0 \\
\hline Min. - Max. & \multicolumn{2}{|c|}{$\begin{array}{c}22.0-46.0 \\
27.47 \pm 5.16\end{array}$} \\
\hline 2-Gender & & \\
\hline Male & 4 & 5.7 \\
\hline Female & 65 & 94.3 \\
\hline 3-Marital status & & \\
\hline Single & 7 & 10.0 \\
\hline Married & 62 & 88.6 \\
\hline Divorced & 0 & 0.0 \\
\hline Widow & 1 & 1.4 \\
\hline 4-EducationalQualification & & \\
\hline Technical Diploma 3 years & 29 & 41.4 \\
\hline Technical Diploma 5 years & 0 & 0.0 \\
\hline Nursing Technician Institute & 10 & 14.3 \\
\hline Institute of technical healthy & 1 & 1.4 \\
\hline Bachelor of nursing & 30 & 42.9 \\
\hline 5-Department & & \\
\hline Neonatal & 0 & 0.0 \\
\hline Emergency & 11 & 15.7 \\
\hline I.C.U & 15 & 21.4 \\
\hline Inpatient & 34 & 48.6 \\
\hline Operation & 10 & 14.3 \\
\hline 6-Years of experiences & & \\
\hline$>5$ & 57 & 81.4 \\
\hline $5-<10$ & 3 & 4.3 \\
\hline $10-<15$ & 4 & 5.7 \\
\hline$\geq 15$ & 6 & 8.6 \\
\hline
\end{tabular}


Table (2): Main score of organizational citizenship behavior dimensions and socio demographic data in general Port Said hospital $(\mathrm{n}=137)$

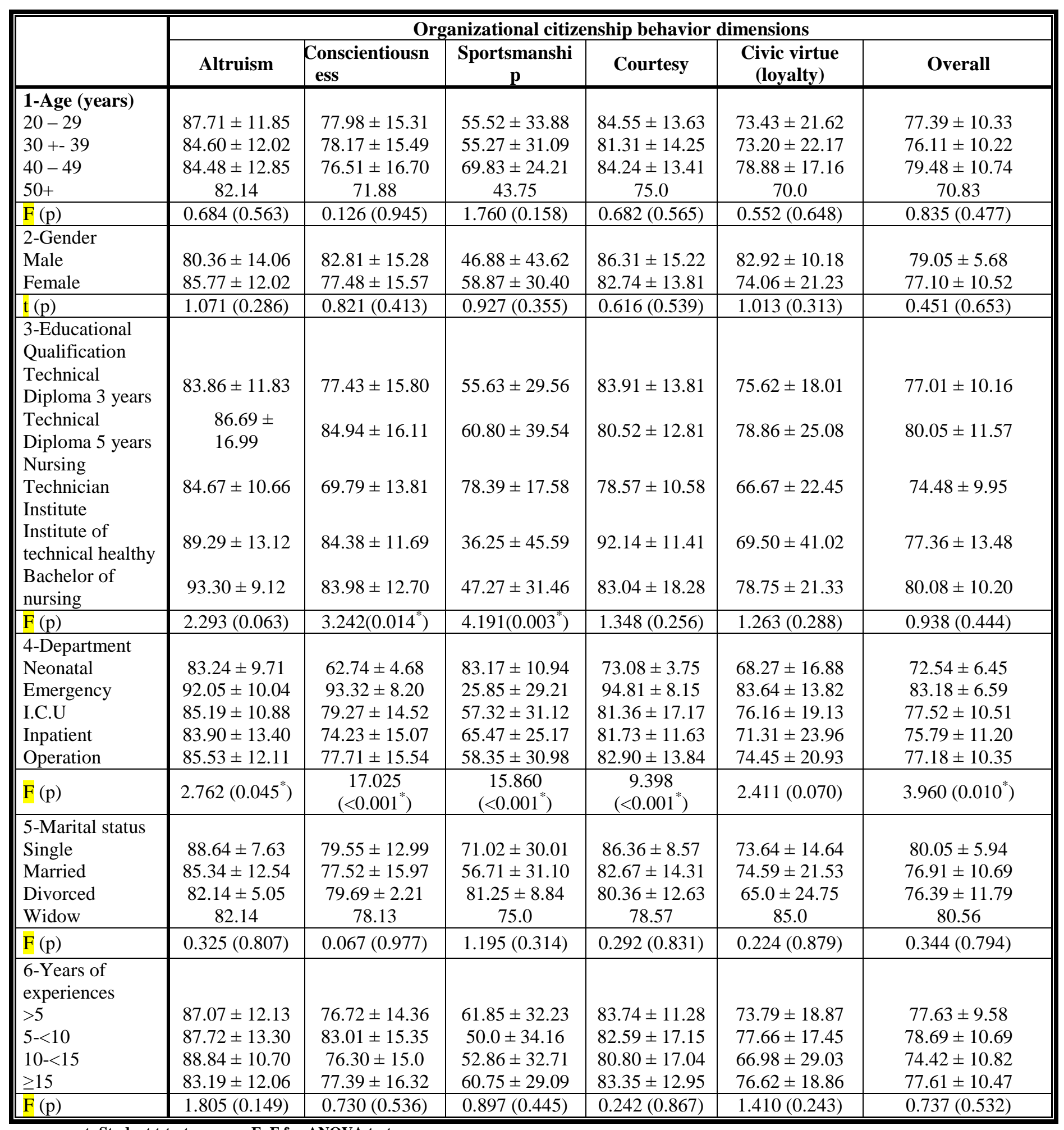

t: Student t-test F: F for ANOVA test

p: $p$ value for comparing between the different categories

*: Statistically significant at $\mathbf{p} \leq \mathbf{0 . 0 5}$

Table (2): Mean score of organizational citizenship behavior dimension and socio demographic data at Atta specialized hospital $(\mathrm{n}=70)$ "continue" 


\begin{tabular}{|c|c|c|c|c|c|c|}
\hline & \multicolumn{6}{|c|}{ Organizational citizenship behavior dimension } \\
\hline & Altruism & $\begin{array}{c}\text { Conscientio } \\
\text { usness }\end{array}$ & \begin{tabular}{|c|}
$\begin{array}{c}\text { Sportsmans } \\
\text { hip }\end{array}$ \\
\end{tabular} & Courtesy & $\begin{array}{c}\text { Civic virtue } \\
\text { (loyalty) }\end{array}$ & Overall \\
\hline \multicolumn{7}{|l|}{ 1-Age (years) } \\
\hline $20-29$ & $74.93 \pm 8.4$ & $69.44 \pm 15.07$ & $46.99 \pm 24.96$ & $71.43 \pm 10.77$ & $73.89 \pm 11.42$ & $69.64 \pm 7.75$ \\
\hline $30+-39$ & $74.11 \pm 7.93$ & $67.19 \pm 11.03$ & $50.52 \pm 20.89$ & $73.51 \pm 9.69$ & $70.63 \pm 12.66$ & $68.87 \pm 6.32$ \\
\hline $40-49$ & $69.64 \pm 4.61$ & $74.22 \pm 13.1$ & $48.44 \pm 36.22$ & $75 \pm 18.9$ & $83.75 \pm 13.77$ & $73.26 \pm 7.78$ \\
\hline $\mathbf{F}(\mathbf{p})$ & $0.793(0.457)$ & $0.365(0.696)$ & 0.100(0.905) & $0.329(0.721)$ & $1.873(0.162)$ & $0.523(0.595)$ \\
\hline \multicolumn{7}{|l|}{ 2-Gender } \\
\hline Male & $75 \pm 10.51$ & $78.91 \pm 8.98$ & $34.38 \pm 28.18$ & $65.18 \pm 11.8$ & $68.75 \pm 11.99$ & $67.71 \pm 6.04$ \\
\hline Female & $74.46 \pm 8.1$ & $68.75 \pm 14.37$ & $48.48 \pm 24.42$ & $72.4 \pm 10.9$ & $74.2 \pm 11.91$ & $69.83 \pm 7.58$ \\
\hline$t(p)$ & $\begin{array}{l}0.128(0.899) \\
\end{array}$ & $1.392(0.169)$ & \begin{tabular}{|l|}
$1.114(0.269)$ \\
\end{tabular} & $1.283(0.204)$ & \begin{tabular}{|l|}
0.889 \\
\end{tabular}$(0.377)$ & $\begin{array}{l}0.549(0.585) \\
\end{array}$ \\
\hline \multirow{2}{*}{\multicolumn{7}{|c|}{$\begin{array}{c}\text { 3EducationalQualificatio } \\
\mathbf{n}\end{array}$}} \\
\hline & & & & & & \\
\hline $\begin{array}{c}\text { Technical Diploma } 3 \\
\text { years }\end{array}$ & $76.85 \pm 10.31$ & $73.28 \pm 12.18$ & $50.43 \pm 21.84$ & $72.41 \pm 12.62$ & $76.12 \pm 10.77$ & $72.05 \pm 7.52$ \\
\hline $\begin{array}{l}\text { Nursing Technician } \\
\text { Institute }\end{array}$ & $72.5 \pm 2.41$ & $63.75 \pm 21.51$ & $38.13 \pm 23.1$ & $69.64 \pm 11.2$ & $68.25 \pm 7.73$ & $65 \pm 7.02$ \\
\hline $\begin{array}{l}\text { Institute of technical } \\
\text { healthy }\end{array}$ & 64.2 & 56.25 & 93.75 & 82.14 & 77.5 & 72.92 \\
\hline Bachelor of nursing & $73.21 \pm 6.48$ & $67.81 \pm 12.77$ & $46.67 \pm 26.55$ & $72.02 \pm 9.43$ & $73.5 \pm 13.77$ & $68.91 \pm 7.01$ \\
\hline $\mathbf{F}(\mathbf{p})$ & $1.833(0.150)$ & $1.692(0.177)$ & $1.873(0.143)$ & $0.440(0.725)$ & $1.138(0.340)$ & $2.617(0.058)$ \\
\hline \multicolumn{7}{|l|}{ 4-Department } \\
\hline Emergency & $69.48 \pm 5.14$ & $\begin{array}{c}71.88 \pm \\
11.35\end{array}$ & $\begin{array}{c}53.98 \pm \\
27.57\end{array}$ & $64.94 \pm 7.11$ & $71.36 \pm 3.6$ & $67.93 \pm 5.41$ \\
\hline I.C.U & $72.38 \pm 5.64$ & $63.54 \pm 17.62$ & $40.42 \pm 20.85$ & $71.43 \pm 13.43$ & $71.33 \pm 15.35$ & $66.39 \pm 7.17$ \\
\hline Inpatient & $76.37 \pm 9.26$ & $72.33 \pm 11.62$ & $49.45 \pm 27.45$ & $74.68 \pm 11.03$ & $76.69 \pm 12.46$ & $72.24 \pm 7.8$ \\
\hline Operation & $76.79 \pm 7.76$ & $65.0 \pm 17.91$ & $45.63 \pm 14.75$ & $71.43 \pm 7.34$ & $71.0 \pm 8.76$ & $68.06 \pm 6.65$ \\
\hline $\mathbf{F}(\mathbf{p})$ & $2.775^{*}\left(0.048^{*}\right)$ & $1.811(0.154)$ & \begin{tabular}{|l|}
$0.747(0.528)$ \\
\end{tabular} & $2.344(0.081)$ & $1.235(0.304)$ & $2.871^{*}\left(0.043^{*}\right)$ \\
\hline \multicolumn{7}{|l|}{ 5-Maritalstatus } \\
\hline Single & $70.41 \pm 4.93$ & $64.29 \pm 15.51$ & $37.5 \pm 20.73$ & $64.29 \pm 6.84$ & $67.5 \pm 3.54$ & $63.39 \pm 3.47$ \\
\hline Married & $750 \pm 8.41$ & $69.96 \pm 14.25$ & $48.19 \pm 24.62$ & $72.98 \pm 11.1$ & $74.6 \pm 12.39$ & $70.4 \pm 7.55$ \\
\hline Widow & 71.43 & 65.63 & 87.5 & 64.29 & 75.0 & 71.53 \\
\hline $\mathbf{F}(\mathbf{p})$ & $1.068(0.349)$ & $0.524(0.594)$ & $1.970(0.147)$ & $2.303(0.108)$ & \begin{tabular}{|l|}
$1.129(0.330)$ \\
\end{tabular} & $2.943(0.060)$ \\
\hline \multicolumn{7}{|l|}{ 6-Years of experiences } \\
\hline$>5$ & $74.44 \pm 8.53$ & $69.08 \pm 14.94$ & $43.42 \pm 22.52$ & $70.8 \pm 10.76$ & $73.6 \pm 11.06$ & $68.86 \pm 7.28$ \\
\hline $5-<10$ & $73.81 \pm 8.99$ & $60.42 \pm 10.05$ & $77.08 \pm 15.73$ & $77.38 \pm 5.46$ & $73.33 \pm 14.22$ & $71.76 \pm 6.67$ \\
\hline $10-<15$ & $80.36 \pm 3.57$ & $70.31 \pm 10.36$ & $73.44 \pm 23.03$ & $79.46 \pm 8.93$ & $68.13 \pm 21.64$ & $73.78 \pm 11.27$ \\
\hline$\geq 15$ & $71.43 \pm 5.05$ & $75.52 \pm 10.54$ & $56.25 \pm 30.62$ & $75.6 \pm 14.71$ & $80.83 \pm 11.58$ & $74.07 \pm 6.16$ \\
\hline$F(p)$ & $0.977(0.409)$ & \begin{tabular}{|l|}
$0.771(0.514)$ \\
\end{tabular} & \begin{tabular}{|c|}
$4.205^{*}\left(0.009^{*}\right)$ \\
\end{tabular} & $1.312(0.278)$ & \begin{tabular}{|c|}
$1.009(0.394)$ \\
\end{tabular} & $1.422(0.244)$ \\
\hline
\end{tabular}


Table (3): Mean score of quality of work life components and socio demographic data in general Port Said hospital $(\mathrm{n}=137)$

\begin{tabular}{|c|c|c|c|c|c|c|c|c|c|c|}
\hline & \multicolumn{10}{|c|}{ Quality of work life components } \\
\hline & $\begin{array}{c}\text { Work } \\
\text { exvironment }\end{array}$ & $\begin{array}{l}\text { Organization } \\
\text { culture }\end{array}$ & $\begin{array}{l}\text { Relation and } \\
\text { cooperation }\end{array}$ & $\begin{array}{l}\text { Training and } \\
\text { development }\end{array}$ & $\begin{array}{l}\text { Compensation } \\
\text { and rewards }\end{array}$ & Facilities & $\begin{array}{c}\text { Job } \\
\text { satisfaction } \\
\text { and job } \\
\text { security }\end{array}$ & $\begin{array}{c}\text { Autonony of } \\
\text { work }\end{array}$ & $\begin{array}{l}\text { Adequacy of } \\
\text { resources }\end{array}$ & Overall \\
\hline 1-Age (years) & & & & & & & & & & \\
\hline $\begin{array}{l}20-29 \\
30+-39 \\
40-49 \\
50+ \\
\end{array}$ & $\begin{array}{c}65.50=21.41 \\
61.85=25.01 \\
67.53=23.92 \\
25.0 \\
\end{array}$ & $\begin{array}{c}73.09=24.59 \\
68.97=29.93 \\
63.30=29.92 \\
57.14\end{array}$ & $\begin{array}{c}67.83=18.95 \\
69.53=19.71 \\
68.97=25.38 \\
41.67 \\
\end{array}$ & $\begin{aligned} & 82.331=24.58 \\
& 79.84=26.46 \\
& 78.97=25.54 \\
& 60.0\end{aligned}$ & $\begin{array}{c}60.70=38.75 \\
58.28=37.95 \\
53.10=37.43 \\
10.0\end{array}$ & $\begin{array}{c}56.28=39.04 \\
55.16=38.50 \\
46.90=38.28 \\
60.0\end{array}$ & $\begin{array}{c}71.93=29.71 \\
62.83=30.04 \\
62.07=27.07 \\
78.57\end{array}$ & $\begin{array}{c}61.82=22.87 \\
61.85=25.96 \\
55.75=26.17 \\
58.33\end{array}$ & $\begin{array}{c}77.13=29.78 \\
67.97=35.92 \\
64.37=36.39 \\
66.67\end{array}$ & $\begin{array}{c}68.28=21.72 \\
65.05=23.68 \\
62.38=25.32 \\
51.0 \\
\end{array}$ \\
\hline$F(p)$ & $1.350(0.261)$ & $0.747(0.526)$ & \begin{tabular}{|c|}
$0.623(0.601)$ \\
\end{tabular} & $0.330(0.804)$ & $0.757(0.520)$ & $0.403(0.751)$ & $1.061(0.368)$ & $0.451(0.717)$ & $0.964(0.412)$ & \begin{tabular}{|l}
$0.507(0.678)$ \\
\end{tabular} \\
\hline \begin{tabular}{|l|} 
2-Gender \\
Male \\
Female \\
\end{tabular} & $\begin{array}{l}73.61=20.69 \\
63.49 \pm 23.90 \\
\end{array}$ & $\begin{array}{l}78.57=26.34 \\
68.54=28.38\end{array}$ & $\begin{array}{l}75.0=12.91 \\
68.38=21.0\end{array}$ & $\begin{array}{c}90.0 \pm 16.73 \\
79.85=25.78\end{array}$ & $\begin{array}{l}61.67=39.20 \\
57.40=38.08\end{array}$ & $\begin{array}{c}65.0=41.35 \\
53.28=38.32\end{array}$ & $\begin{array}{l}86.90=17.15 \\
64.67=29.47\end{array}$ & $\begin{array}{l}68.06=16.17 \\
60.18=25.24\end{array}$ & $\begin{array}{l}91.67=13.94 \\
69.08=34.54\end{array}$ & $\begin{array}{l}76.33=11.33 \\
64.89=23.62\end{array}$ \\
\hline$t(p)$ & $1.020(0.310)$ & $0.849(0.397)$ & $0.763(0.447)$ & $0.954(0.342)$ & $0.268(0.789)$ & $0.730(0.467)$ & $1.830(0.069)$ & $0.756(0.451)$ & $3.505(0.008)$ & $2.259(0.057)$ \\
\hline \begin{tabular}{|l|} 
3EducationalQuali \\
fication \\
Technical Diploma \\
3 years \\
Technical Diploma \\
5 years \\
Nursing Technician \\
Institute \\
Institute of \\
technical healthy \\
Bachelor of nursing
\end{tabular} & $\begin{array}{l}64.61=23.18 \\
62.12=23.08 \\
63.54=26.56 \\
63.33=29.81 \\
62.50=24.15\end{array}$ & $\begin{array}{l}66.58=30.95 \\
79.87=24.29 \\
65.48=22.85 \\
82.86=19.95 \\
74.55=24.60\end{array}$ & $\begin{array}{l}68.0=22.07 \\
77.27=17.52 \\
67.71=20.61 \\
60.0=19.90 \\
70.31=15.80\end{array}$ & $\begin{array}{l}82.96=21.76 \\
74.55=42.28 \\
76.67=27.45 \\
68.0=38.34 \\
80.0=22.21\end{array}$ & $\begin{array}{l}62.84=37.56 \\
57.27=40.52 \\
38.75=34.55 \\
44.0=42.78 \\
63.75=36.49\end{array}$ & $\begin{array}{c}60.62=37.16 \\
60.91=39.36 \\
34.17=33.48 \\
50.0=50.0 \\
45.0=39.67\end{array}$ & $\begin{array}{l}68.34=26.46 \\
68.83=33.39 \\
59.52=32.13 \\
70.0=44.72 \\
57.59=32.21\end{array}$ & $\mid \begin{array}{l}62.65=25.65 \\
74.24=18.80 \\
50.69=22.10 \\
58.33=18.63 \\
55.73=26.48\end{array}$ & $\begin{array}{l}70.37=34.66 \\
80.30=25.62 \\
70.14=34.75 \\
90.0=22.36 \\
55.21=36.37\end{array}$ & $\begin{array}{l}67.19=24.19 \\
70.55=21.67 \\
58.50=20.89 \\
64.80=24.51 \\
63.31=23.42\end{array}$ \\
\hline $\mathrm{F}(\mathrm{p})$ & $0.048(0.996)$ & $1.106(0.356)$ & $0.746(0.562)$ & $0.769(0.547)$ & $2.203(0.072)$ & $2.650(0.036)$ & $0.788(0.535)$ & $2.142(0.079)$ & $1.447(0.222)$ & $0.807(0.523)$ \\
\hline $\begin{array}{l}\text { 4-Department } \\
\text { Neonatal } \\
\text { Emergency } \\
\text { I.C.U } \\
\text { Inpatient }\end{array}$ & $\begin{array}{l}79.49=23.72 \\
71.21=16.0 \\
62.80=23.58 \\
58.74=24.69\end{array}$ & $\begin{array}{l}79.67=39.11 \\
80.19=23.53 \\
66.38=22.83 \\
64.40=29.46\end{array}$ & $\begin{array}{l}75.0=30.99 \\
69.32=15.93 \\
70.33=16.36 \\
65.98=22.27\end{array}$ & $\begin{array}{l}87.69=24.21 \\
93.64=11.36 \\
78.78=22.72 \\
74.92=29.30\end{array}$ & $\begin{array}{l}79.23=39.68 \\
83.18=30.14 \\
52.44=34.91 \\
47.21=36.84\end{array}$ & $\begin{array}{l}79.23=39.68 \\
80.45=28.53 \\
48.29=35.77 \\
42.46=36.50\end{array}$ & $\begin{array}{l}75.82=20.32 \\
85.39=16.71 \\
60.98=27.16 \\
59.48=32.60\end{array}$ & $\begin{array}{l}81.41=35.54 \\
75.76=10.88 \\
52.03=22.88 \\
56.28=22.75\end{array}$ & $\begin{array}{l}79.49=39.76 \\
94.70=13.0 \\
64.23=34.07 \\
63.11=34.44 \\
\end{array}$ & $\begin{array}{c}79.46=31.39 \\
80.55=9.73 \\
61.85=21.90 \\
59.31=22.56 \\
\end{array}$ \\
\hline $\mathrm{F}(\mathrm{p})$ & $3.753(0.013)$ & $2.502(0.062)$ & $0.837(0.476)$ & $3.510(0.017)$ & $7.423(<0.001)$ & $8.789(<0.001)$ & $5.595(0.001)$ & $9.439(<0.001)$ & $5.963(0.001)$ & $7.254(<0.001)$ \\
\hline $\begin{array}{l}\text { 5-Marital status } \\
\text { Single } \\
\text { Married } \\
\text { Divorcad } \\
\text { Widow } \\
\end{array}$ & $\begin{array}{c}62.88=20.19 \\
64.09=24.24 \\
54.17 \pm 29.46 \\
75.0\end{array}$ & $\begin{aligned} & 65.58=24.50 \\
& 69.22=28.69 \\
& 67.86=45.46 \\
& 78.57\end{aligned}$ & $\begin{aligned} & 65.15=20.01 \\
& 69.38=20.76 \\
& 54.17=29.46 \\
& 50.0\end{aligned}$ & $\begin{array}{c}81.82=26.76 \\
80.57=25.33 \\
70.0=42.43 \\
50.0\end{array}$ & $\begin{array}{c}53.64=39.82 \\
58.29=38.02 \\
40.0=56.57 \\
50.0\end{array}$ & $\begin{array}{c}42.73=38.49 \\
55.61=38.35 \\
30.0=14.14 \\
0.0\end{array}$ & $\begin{array}{c}71.43=28.75 \\
65.45=29.41 \\
60.71=45.46 \\
35.71\end{array}$ & $\begin{array}{c}56.06=29.60 \\
60.98=24.78 \\
66.67=11.79 \\
41.67\end{array}$ & $\begin{aligned} & 65.15=34.52 \\
& 70.87=34.18 \\
& 58.33=58.93 \\
& 50.0\end{aligned}$ & $\begin{array}{c}63.0=21.62 \\
65.89=23.51 \\
56.50=36.06 \\
49.0\end{array}$ \\
\hline$F(p)$ & $0.190(0.903)$ & $0.093(0.964)$ & $0.746(0.526)$ & $0.592(0.621)$ & $0.206(0.892)$ & $1.318(0.271)$ & $0.504(0.680)$ & \begin{tabular}{|l|}
$0.357(0.784)$ \\
\end{tabular} & $0.287(0.835)$ & $0.314(0.815)$ \\
\hline $\begin{array}{l}\text { 6-Years of } \\
\text { experiences } \\
>5 \\
5<10 \\
10<15 \\
\geq 15\end{array}$ & $\begin{array}{l}66.09=19.41 \\
68.23=22.20 \\
48.61=23.14 \\
67.40=48.61\end{array}$ & $\begin{array}{l}70.94=22.82 \\
79.02=23.25 \\
65.77=33.50 \\
66.91=29.41\end{array}$ & $\begin{array}{l}70.69=18.85 \\
62.50=16.94 \\
63.89=21.93 \\
70.96=21.67\end{array}$ & $\begin{array}{l}82.41=23.55 \\
86.88=18.87 \\
73.33=29.73 \\
80.29=25.97\end{array}$ & $\begin{array}{l}61.38=36.81 \\
61.88=40.20 \\
47.50=40.24 \\
58.53=37.39\end{array}$ & $\begin{array}{l}54.83=35.82 \\
61.25=44.55 \\
37.92=36.95 \\
57.21=37.73\end{array}$ & $\begin{array}{l}73.65=28.19 \\
70.54=28.44 \\
45.54=29.47 \\
68.17=27.44\end{array}$ & $\begin{array}{l}60.06 \pm 22.54 \\
70.31=21.07 \\
52.43=26.86 \\
61.27=25.58\end{array}$ & $\begin{array}{l}73.56=29.72 \\
79.17=34.16 \\
52.78=38.91 \\
72.55=32.93\end{array}$ & $\begin{array}{l}68.14=20.65 \\
70.81=21.26 \\
54.42=23.76 \\
66.82=23.97\end{array}$ \\
\hline $\mathbf{F}(\mathrm{p})$ & $4.354(0.006)$ & $0.941(0.423)$ & $1.274(0.286)$ & $1.019(0.386)$ & \begin{tabular}{|c|}
$0.738(0.531)$ \\
\end{tabular} & $1.788(0.153)$ & $5.235(0.002)$ & $1.717(0.167)$ & $2.746(0.046)$ & $2.349(0.075)$ \\
\hline
\end{tabular}

F: F for ANOVA test

t: Student t-test

p: $p$ value for associated between different categories

$*$ : Statistically significant at $\mathbf{p} \leq \mathbf{0 . 0 5}$ 
Table (3): Mean score of quality of work life components and socio demographic data at

Atta specialized hospital $(\mathrm{n}=70)$ "continue"

\begin{tabular}{|c|c|c|c|c|c|c|c|c|c|c|}
\hline & \multicolumn{10}{|c|}{ Quality of work life components } \\
\hline & $\begin{array}{c}\text { Work } \\
\text { environment }\end{array}$ & $\begin{array}{l}\text { Organization } \\
\text { culture }\end{array}$ & $\begin{array}{c}\text { Relation and } \\
\text { cooperation }\end{array}$ & $\begin{array}{l}\text { Training and } \\
\text { development }\end{array}$ & $\begin{array}{c}\text { Compensatio } \\
\text { n and } \\
\text { rewards }\end{array}$ & Facilities & \begin{tabular}{|c|} 
Job \\
satisfaction \\
and job \\
security
\end{tabular} & $\begin{array}{c}\text { Autonomy of } \\
\text { work }\end{array}$ & $\begin{array}{c}\text { Adequacy of } \\
\text { resources }\end{array}$ & Overall \\
\hline \begin{tabular}{||l|}
$1-$ Age (years) \\
$20-29$ \\
$30+-39$ \\
$40-49$ \\
F(p) \\
\end{tabular} & $\begin{array}{l}67.59=13.51 \\
56.25=17.45 \\
66.67=13.61 \\
3.128^{2}\left(0.050^{7}\right)\end{array}$ & $\begin{array}{c}63.1=17.76 \\
63.69=21.2 \\
76.79=15.84 \\
1.045(0.357) \\
\end{array}$ & $\begin{array}{c}65.9=20.56 \\
70.14=17.93 \\
75=16.67 \\
0.549(0.580) \\
\end{array}$ & $\begin{array}{l}68.15=28.22 \\
61.67=29.18 \\
82.5=23.63 \\
0.832(0.440) \\
\end{array}$ & $\mid \begin{array}{c}59.63=23.18 \\
55.83=28.43 \\
65=23.8 \\
0.241(0.787) \\
\end{array}$ & $\mid \begin{array}{c}55.93=27.44 \\
62.5=31.66 \\
60=27.08 \\
0.287(0.751) \\
\end{array}$ & $\begin{array}{l}64.55=22.19 \\
67.26=25.79 \\
66.07=28.2 \\
0.071(0.931) \\
\end{array}$ & $\begin{array}{l}63.73=12.6 \\
62.5=20.26 \\
60.42=18.48 \\
0.122(0.885)\end{array}$ & $\begin{array}{l}63.58=24.0 \\
66.67=32.57 \\
58.33=28.87 \\
0.165(0.848)\end{array}$ & \begin{tabular}{|c|}
$63.72=11.19$ \\
$63=17.36$ \\
$68.5=17.82$ \\
$0.295(0.745)$ \\
\end{tabular} \\
\hline \begin{tabular}{|c|}
$\begin{array}{c}\text { 2-Gender } \\
\text { Male } \\
\text { Female }\end{array}$ \\
$t(\mathrm{p})$ \\
\end{tabular} & $\begin{array}{c}60.42=10.49 \\
65.91=14.89 \\
0.725(0.471) \\
\end{array}$ & $\begin{array}{c}66.07=10.71 \\
63.85=18.71 \\
0.234(0.816) \\
\end{array}$ & $\begin{array}{c}68.75=17.18 \\
67.05=20.12 \\
0.166(0.869) \\
\end{array}$ & $\begin{array}{c}75.0=17.32 \\
67.42=28.68 \\
0.520(0.605) \\
\end{array}$ & \begin{tabular}{|l|}
$75.0=19.15$ \\
$58.33=23.9$ \\
$1.365(0.177)$
\end{tabular} & \begin{tabular}{|c|}
$82.5=15.0$ \\
$55.76=27.79$ \\
$1.898(0.062)$ \\
\end{tabular} & \begin{tabular}{|l|}
$66.07=23.6$ \\
$65.04=22.9$ \\
$0.087(0.931)$ \\
\end{tabular} & \begin{tabular}{|l|}
$75.0=9.62$ \\
$62.63=14.27$ \\
$1.705(0.093)$ \\
\end{tabular} & $\begin{array}{c}62.5=25 \\
63.89=25.75 \\
0.105(0.917) \\
\end{array}$ & \begin{tabular}{|l|}
$70.0=14.67$ \\
$63.5=12.52$ \\
$1.000(0.321)$ \\
\end{tabular} \\
\hline \begin{tabular}{||l|} 
E- \\
EducationalQualif \\
cation \\
Technical Diploma \\
3 years \\
Nursing Technician \\
Institute \\
Institute of technical \\
healthy \\
Bachelor of nursing \\
$F(p)$ \\
\end{tabular} & $\begin{array}{c}70.11=13.27 \\
60.83=12.45 \\
75.0 \\
62.5=15.89 \\
1.925(0.134)\end{array}$ & $\begin{array}{c}65.27=19.16 \\
52.14=20.5 \\
64.29 \\
66.67=15.95 \\
1.710(0.174)\end{array}$ & $\begin{array}{c}72.99=17.06 \\
54.17=14.83 \\
83.3 \\
65.28=22 \\
2.769^{2}(0.049)\end{array}$ & $\begin{array}{c}71.72=26.06 \\
60=38.59 \\
90.0 \\
66=26.6 \\
0.684(0.565)\end{array}$ & $\mid \begin{array}{c}61.03=26.91 \\
48=13.98 \\
50.0 \\
61.67 \pm 23.21 \\
0.946(0.424)\end{array}$ & $\begin{array}{c}61.38=27.35 \\
47 \pm 26.69 \\
30.0 \\
57.67=28.73 \\
0.983(0.406)\end{array}$ & $\mid \begin{array}{c}70.69=25.29 \\
55.71=15.36 \\
64.29 \\
62.86=21.83 \\
1.261(0.295)\end{array}$ & $\left\{\begin{array}{c}67.53=12.6 \\
54.17=15.84 \\
50.0 \\
62.78=14.14 \\
2.697(0.053)\end{array}\right.$ & $\begin{array}{c}64.94=23.29 \\
71.67=23.64 \\
50.0 \\
60.56=28.5 \\
0.584(0.628)\end{array}$ & $\begin{array}{c}67.62=10.99 \\
55.2=9.54 \\
63.0 \\
63.17=13.92 \\
2.633(0.057) \\
\end{array}$ \\
\hline \begin{tabular}{|l|} 
4-Department \\
Emergency \\
I.C.U \\
Inpatient \\
Operation \\
F(p)
\end{tabular} & $\begin{array}{l}64.39=13.48 \\
62.78=16.33 \\
65.2=15.14 \\
72.5=11.15 \\
0.954(0.420)\end{array}$ & $\begin{array}{r}64.29=7.14 \\
64.29=19.09 \\
64.29=19.97 \\
62.14=21.83 \\
0.038(0.990)\end{array}$ & $\begin{array}{l}69.7=19.46 \\
54.44=21.56 \\
71.08=17.68 \\
70.0=19.72 \\
2.828^{2}(0.045)\end{array}$ & $\begin{array}{c}76.36=19.63 \\
52.67=38.82 \\
71.76=21.24 \\
68.0=33.27 \\
2.110(0.107)\end{array}$ & \begin{tabular}{|l}
$54.55=5.22$ \\
$54.0=22.61$ \\
$61.47=25.99$ \\
$65.0=30.64$ \\
$0.667(0.575)$
\end{tabular} & $\begin{array}{c}50=14.83 \\
47.33=30.81 \\
60.59=27.3 \\
69.0=32.81 \\
1.685(0.179)\end{array}$ & $\begin{array}{l}61.69=18.43 \\
51.43=17.54 \\
70.59=23.9 \\
70.71=23.19 \\
2.983^{2}(0.037\end{array}$ & $\begin{array}{c}64.39=9.2 \\
58.33=15.11 \\
65.2=15.82 \\
63.33=11.92 \\
0.819(0.488)\end{array}$ & $\begin{array}{l}53.03=16.3 \mathrm{~d} \\
67.78=31.79 \\
59.31=23.64 \\
85.0=18.34 \\
3.845(0.013\end{array}$ & $\begin{array}{c}62.73=3.74 \\
56.73=14.49 \\
66.0=11.66 \\
68.6=15.88 \\
2.585(0.061)\end{array}$ \\
\hline \begin{tabular}{|l|l} 
5-Marital status \\
Single \\
Married \\
Widow \\
\end{tabular} & $\begin{array}{c}59.52=13.97 \\
66.26=14.82 \\
66.67\end{array}$ & $\begin{array}{c}55.1=12.85 \\
64.98=18.77 \\
64.29\end{array}$ & $\begin{array}{c}51.19=16.96 \\
68.68=19.54 \\
83.33\end{array}$ & $\begin{array}{c}65.71=31.55 \\
67.58=27.92 \\
100.0\end{array}$ & $\begin{array}{c}55.71=22.99 \\
59.84=24.26 \\
50.0\end{array}$ & $\begin{array}{c}65.71=23.7 \\
56.61=28.45 \\
40.0\end{array}$ & $\begin{array}{c}55.1=20.5 \\
66.01=23.02 \\
78.57\end{array}$ & $\left\{\begin{array}{c}61.9=15.11 \\
63.71=14.32 \\
50.0\end{array}\right.$ & $\left\{\begin{array}{c}73.81=23.29 \\
62.9=25.86 \\
50.0\end{array}\right.$ & $6 \begin{array}{c}59.29=15.38 \\
64.35=12.42 \\
66.0\end{array}$ \\
\hline \begin{tabular}{|c|}
$\mathrm{F}(\mathrm{p})$ \\
6-Years of
\end{tabular} & $0.660(0.520)$ & $0.914(0.406)$ & $2.933(0.060)$ & $0.670(0.515)$ & $0.167(0.847)$ & $0.523(0.595)$ & $0.897(0.413)$ & $0.485(0.618)$ & $0.716(0.492)$ & $0.514(0.600)$ \\
\hline $\begin{array}{l}>5 \\
5<10 \\
10<15 \\
\geq 15\end{array}$ & $\begin{array}{c}66.96=13.36 \\
50=22.05 \\
52.08=21.92 \\
69.44=12.55\end{array}$ & $\begin{array}{c}62.66=18.36 \\
54.76=8.25 \\
69.64=16.88 \\
77.38=18.3\end{array}$ & $\begin{aligned} & 64.77=20.29 \\
& 80.56=4.81 \\
& 77.08=21.92 \\
& 76.39=13.35 \\
&\end{aligned}$ & $\begin{array}{c}65.44=29.4 \\
73.33=15.28 \\
72.5=22.17 \\
85=19.75\end{array}$ & $\begin{array}{c}58.6=20.83 \\
30=26.46 \\
65=43.59 \\
76.67=25.82\end{array}$ & $\begin{array}{l}55.09=27.33 \\
33.33=5.77 \\
82.5=20.62 \\
73.33=29.44\end{array}$ & $\begin{array}{l}62.41=22.04 \\
66.67=17.98 \\
83.93=20.52 \\
77.38=28\end{array}$ & $\begin{array}{l}64.33=12.48 \\
38.89=26.79 \\
60.42=7.98 \\
68.06=18.5\end{array}$ & $\begin{array}{l}63.74=23.81 \\
50.0=50.0 \\
62.5=28.46 \\
72.22=31.03\end{array}$ & $\left\{\begin{array}{c}62.77=10.97 \\
54=13.89 \\
70=18.71 \\
75.17=17.59\end{array}\right.$ \\
\hline$F(p)$ & $2.759^{2}\left(0.049^{\prime}\right)$ & $1.593(0.199)$ & $1.531(0.215)$ & $0.956(0.419)$ & $2.879^{\circ}(0.043$ & $2.818^{\prime}(0.046)$ & $1.828(0.151)$ & $3.677(0.016$ & $0.502(0.682)$ & $2.891^{\circ}(0.042)$ \\
\hline
\end{tabular}

F: F for ANOVA test $\quad$ : $p$ value for associated between different categories

*: Statistically significant at $\mathbf{p} \leq \mathbf{0 . 0 5}$

t: Student t-test * 
Table (4): Comparison between the studied hospitals according to organizational citizenship behavior dimensions

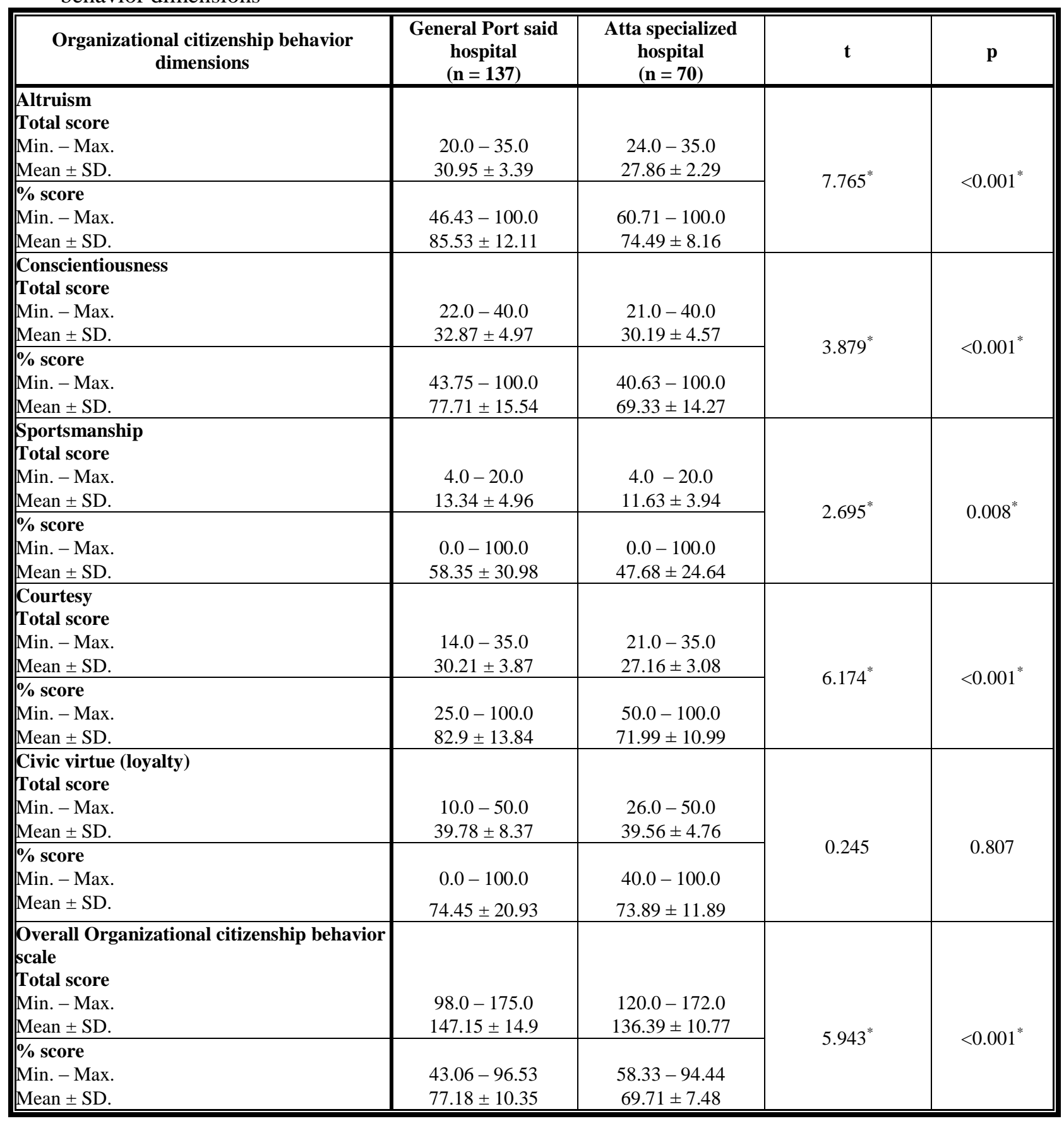

$\mathrm{t}$ : Student t-test

$\mathrm{p}$ : $\mathrm{p}$ value for comparing between the studied hospitals

*: Statistically significant at $\mathrm{p} \leq 0.05$ 
Table (5): Comparison between the studied hospitals according to quality of work life components

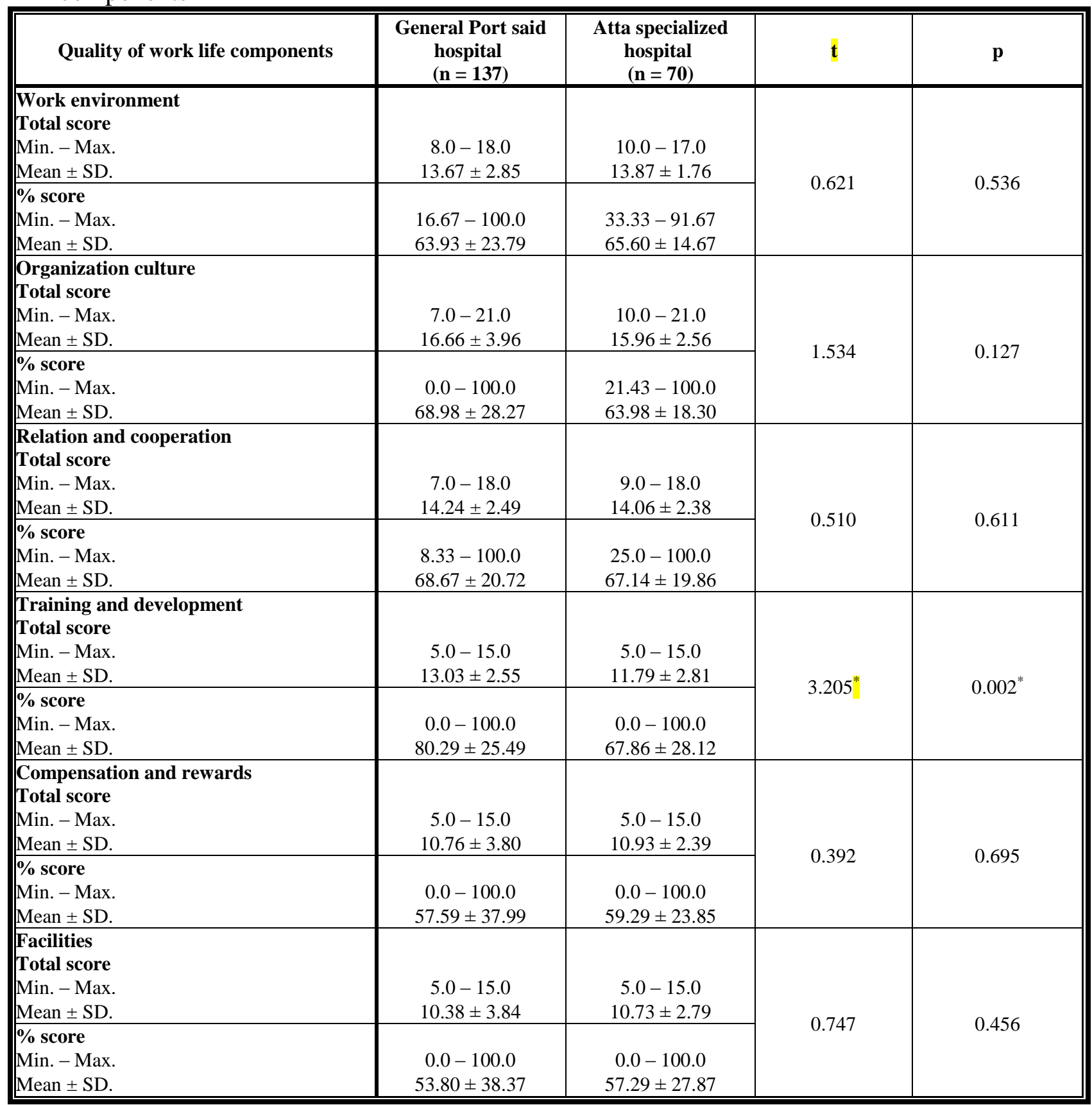

t: Student t-test

$\mathrm{p}$ : $\mathrm{p}$ value for comparing between the studied hospitals

*: Statistically significant at $\mathrm{p} \leq 0.05$ 
Table(5): Comparison between the studied hospitals according to quality of work life components "continue"

\begin{tabular}{|c|c|c|c|c|}
\hline Quality of work life components & $\begin{array}{c}\text { General Port said } \\
\text { hospital } \\
(\mathbf{n}=\mathbf{1 3 7})\end{array}$ & $\begin{array}{l}\text { Atta specialized } \\
\text { hospital } \\
(\mathbf{n}=70)\end{array}$ & $\mathbf{t}$ & $\mathbf{p}$ \\
\hline $\begin{array}{l}\text { Job satisfaction and job security } \\
\text { Total score } \\
\text { Min. - Max. } \\
\text { Mean } \pm \text { SD. }\end{array}$ & $\begin{array}{c}7.0-21.0 \\
16.19 \pm 4.11\end{array}$ & $\begin{array}{c}10.0-21.0 \\
16.11 \pm 3.19\end{array}$ & \multirow{2}{*}{0.146} & \multirow{2}{*}{0.884} \\
\hline $\begin{array}{l}\text { \% score } \\
\text { Min. }- \text { Max. } \\
\text { Mean } \pm \text { SD. }\end{array}$ & $\begin{array}{c}0.0-100.0 \\
65.64 \pm 29.36 \\
\end{array}$ & $\begin{array}{l}21.43-100.0 \\
65.10 \pm 22.77 \\
\end{array}$ & & \\
\hline \begin{tabular}{|l|} 
Autonomy of work \\
Total score \\
Min. - Max. \\
Mean \pm SD. \\
\end{tabular} & $\begin{array}{c}6.0-18.0 \\
13.26 \pm 2.99\end{array}$ & $\begin{array}{c}7.0-16.0 \\
13.60 \pm 1.71\end{array}$ & \multirow{2}{*}{1.030} & \multirow{2}{*}{0.304} \\
\hline $\begin{array}{l}\text { \% score } \\
\text { Min. }- \text { Max. } \\
\text { Mean } \pm \text { SD. }\end{array}$ & $\begin{array}{c}0.0-100.0 \\
60.52 \pm 24.92\end{array}$ & $\begin{array}{c}8.33-83.33 \\
63.33 \pm 14.29\end{array}$ & & \\
\hline $\begin{array}{l}\text { Adequacy of resources } \\
\text { Total score } \\
\text { Min. - Max. } \\
\text { Mean } \pm \text { SD. } \\
\end{array}$ & $\begin{array}{c}3.0-9.0 \\
7.20 \pm 2.05\end{array}$ & $\begin{array}{c}3.0-9.0 \\
6.83 \pm 1.53\end{array}$ & \multirow{2}{*}{1.483} & \multirow{2}{*}{0.140} \\
\hline $\begin{array}{l}\text { \% score } \\
\text { Min. }- \text { Max. } \\
\text { Mean } \pm \text { SD. }\end{array}$ & $\begin{array}{c}0.0-100.0 \\
70.07 \pm 34.19\end{array}$ & $\begin{array}{c}0.0-100.0 \\
63.81 \pm 25.53\end{array}$ & & \\
\hline $\begin{array}{l}\text { Overall quality of work life components } \\
\text { Total score } \\
\text { Min. - Max. } \\
\text { Mean } \pm \text { SD. }\end{array}$ & $\begin{array}{c}56.0-146.0 \\
115.39 \pm 23.31 \\
\end{array}$ & $\begin{array}{c}87.0-144.0 \\
113.87 \pm 12.62\end{array}$ & \multirow{2}{*}{0.609} & \multirow{2}{*}{0.543} \\
\hline $\begin{array}{l}\text { \% score } \\
\text { Min. }- \text { Max. } \\
\text { Mean } \pm \text { SD. }\end{array}$ & $\begin{array}{c}6.0-96.0 \\
65.39 \pm 23.31\end{array}$ & $\begin{array}{c}37.0-94.0 \\
63.87 \pm 12.62\end{array}$ & & \\
\hline
\end{tabular}

t: Student t-test $\mathrm{p}$ : $\mathrm{p}$ value for comparing between the studied hospitals*: Statistically significant at $\mathrm{p} \leq 0.05$

Table (6): Correlation between organizational citizenship behavior dimensions and quality of work life components in total sample $(n=207)$

\begin{tabular}{|c|c|c|}
\hline \multirow{2}{*}{ Quality of work life components } & \multicolumn{2}{|c|}{$\begin{array}{c}\text { Overall organizational } \\
\text { citizenship behavior } \\
\text { dimensions }\end{array}$} \\
\cline { 2 - 3 } & $\mathbf{r}$ & $\mathbf{p}$ \\
\hline Overall quality of work life components & $0.535^{*}$ & $<0.001^{*}$ \\
\hline
\end{tabular}

r:Pearson coefficient

*: Statistically significant at $\mathrm{p} \leq 0.05$ 


\section{DISCUSSION:}

The study findings revealed that there was a strong statistically significant relation between organizational citizenship behavior scale and quality of work life. In the current study, as a baseline for exploring the relationship between organizational citizenship behavior and quality of work life, some great significant results were detected, and this indicates that this protocol is of great value.

Beginning with socio-demographic characteristics in General Port Said hospital as a baseline for the study, it was shown that about half of the studied sample aged $30-39$ years old. Age's mean \pm S.D was $33.12 \pm 7.43$. Most of them were married females, about half of them studied technical diploma 3 years; inpatient department, more than half of them had 15 years' experience and more. On the other side, socio-demographic characteristics at Atta specialized hospital, it was shown that above half of the studied sample aged $20-29$ years old. Age's mean \pm S.D was $27.47 \pm 5.16$. Above half of them were married females, about half of them studied bachelor of nursing; inpatient department, more than half of them had $>5$ years' experience.

This was similar to a study finding by Kazemipour, Amin \&Pourseidi, (2012), as the majority of respondents were married female. Almost half of their experience ranged from 11 to 30 years.

Organizational citizenship behavior scale and socio demographic data in general Port Said hospitalthere were a statistically significant relations to department but there were no statistically significant relations to age (years), gender, educational qualification, marital status and years of experiences. However, relation between organizational citizenship behavior scale and socio demographic data at Atta specialized hospital there were a statistically significant relations to department, but there were no statistically significant relations to age (years), gender, educational qualification, marital status and years of experiences.

A cross-sectional study by(Soto, John, Gosling \& Potter, 2011)studied the five domains of personality and detected that altruism showed age trends similar to conscientiousness, and might be influenced by similar social and biological factors .On the other hand, demographic variables (age, gender, educational qualification, and marital 
status) in the study by (Bakhshi, Sharma\& Kumar, 2011) showed no significant impact on the organizational citizenship behavior.

Quality of work life components and socio demographic data in general Port Said hospital there were no statistically significant relations to age (years), gender, educational qualification, marital status and years of experiences. The only statistically significant relation was found to department. While relation between quality of work life components and socio demographic data at Atta specialized hospital there was no statistically significant relations to age (years), gender, educational qualification, department and marital status. The only statistically significant relation was found to years of experiences.

Egyptian study by (Shazly\&Fakhry, 2014) illustrated that nurses' perception of QWL was higher with increasing age and experience years. This might be explained by the fact that as the nurses get older in age and gain more years of experience, they advance in their career and have higher job status, which has a positive reflection on their QWL. They also highlighted that mature nurses have greater job satisfaction, productivity and organizational commitment. This may be attributed to the ability of older nurses to make a better adjustment to the work environment when compared with younger nurses.

Comparison between the studied hospitals according to organizational citizenship behavior scale: Regarding altruism, conscientiousness, sportsmanship, courtesy, civic virtue and overall organizational citizenship behavior scale there were strongly agreed in general Port said hospital with Atta specialized hospital.

The study by (Jahani M.A.2018), there was a significant relationship between the hospitals in the component "sportsmanship" $(\mathrm{p}=0.05)$. The highest difference was in the relationship between private vs. other (University and social secure)hospitals. The differences in other components were not significant.

Comparison between the studied hospitals according to quality of work life components: Regarding organization culture, relation and cooperation and training and development, job satisfaction and job security, adequacy of resources and overall quality of work life components in general Port said hospital there were more agreed 
with Atta specialized hospital, while work environment, autonomy of work, compensation and rewards and facilities at Atta specialized hospital there were more agreed with general port said hospital.

The study by (Suresh. D.2013), the Quality of Nursing Work Life (QNWL) among nurses working in government and private hospitals are significantly different .Even though nurses in both government and private sector reported a moderate QNWL, the nurses in the government sector had better QNWL than the private sector overall and all the across four domains.

By testing the correlation between organizational citizenship behavior scale and quality of work life, it was detected that the overall organizational citizenship behavior scale is statistically significant correlated to all items of quality of work life components for each of them.

This was on line with the study by (Nafei, 2015) who found an existence of a positive correlation between QWL and OCB implying that the high level of QWL led to high level of OCB. Conversely, (Manaois, 2014) in his study showed that only altruism and civic virtue were associated to QWL, and they were not significant to be predictors of safe and healthy working environment.

A study by Sandhya Nair, (2013) proved significant relationship between the quality of Work Life and OCB based on Altruism. Meta-analyses have found that conscientiousness is related to supervisory ratings of job performance and improved QWL across occupational groups. In addition ,Marzuki (2013) found that conscientiousness was related positively to the service performance and improves QWL. while this correlation is significant at the 0.01 level.

In the study done by Alfonso, Zenasni, Hodzic\& Ripoll, (2016)they stated that QWL also seems to have a positive effect on citizenship at work. The results from this study confirm that the better their quality of work life, the more the workers tend to express citizenship behaviors toward the organization. This effect is due to the sportsmanship dimension, which appears to be affected by quality of work life score, also showed that among the four OCBs, job satisfaction predicted only sportsmanship. 
On completing, courtesy \& civic virtue (loyalty), were significantly correlated to all items of quality of work life; work environment, organization culture, training and development, facilities, job satisfaction and job security, autonomy of work, adequacy of resources, and overall quality of work life components for each of them and also were significantly correlated to compensation and rewards.

The study by Swaminathan\&Jawahar, (2013) proved a positive relationship has been established between courtesy and QWL. In the analysis of the organizational citizenship behaviors and quality of work-life of employees by (Manaois, 2014), civic virtue is a predictor of employees' opportunity for career growth. With the regression, it indicated a significant but weak correlation with having a safe and healthy working condition in the organization.

\section{CONCLUSION:}

In the light of the findings of the current study, it can be concluded that: Organizational Citizenship Behavior (OCB) is affected by Quality of Work Life (QWL). Also, this study finds a positive correlation among organizational citizenship behavior (OCB) and dimensions of Quality of Work Life (QWL). There are significant and direct relationships between organizational citizenship behaviors. The two major components of OCB are compliance, i.e., the employees' intention to follow the organizational rules, and altruism or pro-social, which means employees' voluntary behaviors to help others while working.

\section{RECOMMENDATIONS:}

Based on the findings of the present study, the following recommendations were suggested:Effort of improving OCB can be done by improving the image of the hospital; Increase training programs in hospitals help nurses to achieve the required skills and improve communication among nurses, providing pamphlets and posters for nurses to increase their awareness about OCB, improve QWL dimensions: job enrichment, supervision system, opportunities for self-development and environmental comfort, increase of supportive staffs as it affects their quality of work life and hence enhances organization citizenship behaviour, effort of improving and can be done by improving OCB dimensions: altruism, conscientiousness, civic virtue, courtesy and sportsmanship. 


\section{REFERENCES:}

Abubakar, A.M., Anasori, E., \&Lasisi, T.T. (2019): Physical attractiveness and managerial favoritism in the hotel industry: The light and dark side of erotic capital. Journal of Hospitality and Tourism Management, 38, 16-26.

Alfonso, L., Zenasni, F., Hodzic, S., \& Ripoll, P. (2016):Understanding the mediating role of quality of work life on the relationship between emotional intelligence and organizational citizenship behaviors. Psychological Reports, 118(1), 107-127.

Allameh, S. M., Sha'baniNaftchali, J., Khaza'ee Paul, J., VericKazemi, R., \&Amani, M. (2014): “The presentation of the relationship structure between perceived spiritual leadership with commitment, organizational citizenship and nurses' performance." The seasonal journal of nursing management. 3(3):52-60.

Amini, A., \&Mortazavi, S. (2013): The impact of the broadcasting mistake management culture in a healthy organization on the quality of the personnel work life. Jclin.Diagn,Res;(7),507-512.

Bakhshi, A., Sharma, A.D., \& Kumar, K. (2011): Organizational commitment as predictor of organizational citizenship behavior. European journal of business and management, 3(4), 78-86.

Chowdhury, R. (2019): A holistic approach to employee engagement. In systems thinking for management consultants (pp. 269-304).Springer, Singapore.

Dargahi , H., Alirezaie, S., \&Shaham, G. (2012): Organizational citizenship behavior among Iranian nurses. Iranian journal of public health, 41(5), 85.

Davoudi, M. (2014): The study relationship between quality of work life and human resource development of teachers. International journal of management and humanity sciences. 3 (1): 1269-80.

Elamin, A. M., \&Tlaiss, H. A. (2015): Exploring the relationship between organizational citizenship behavior and organizational justice in the Islamic Saudi Arabian context. Employee Relations, 37(1), 2-29. 
Hamed, A. (2014): Perception of assistant teaching staff of their leaders transformational leadership behaviors and its relation to their organizational citizenship behaviors in Suez Canal University.

Jahani M.A.(2018): Datasets on organizational citizenship behavior in the selected hospitals with different ownership. Journal of health management,(19),288292.

Kazemipour, F., Amin, S. M., \&Pourseidi, B. (2012): Relationship between workplace spirituality and organizational citizenship behavior among nurses through mediation of affective organizational commitment. Journal of Nursing Scholarship, 44(3), 302-310.

Lee, K., \& Allen, N.J. (2002): Organizational citizenship behavior and workplace deviance: the role of affect and cognition. Journal of Applied Psychology, 87:131-42.

Manaois, J. O. (2014): An analysis of the organizational citizenship behaviors and quality of work-life of slers employees (Doctoral dissertation, Xavier University).

Marzuki, N.A. (2013): The impact of personality on employee wellbeing. European Scientific Journal, ESJ, 9(20).

Nafei, W. (2015): Meta-analysis of the impact of psychological capital on quality of work life and organizational citizenship behavior: A study on Sadat City University. International Journal of Business Administration, 6(2), 42.

Podsakoff, M.(1990):Transformational leader behaviors and their effects on followers' trust in leader, satisfaction, and organizational citizenship behaviors. Leadership Quarterly, 1: 107-42

Pradhan, R. K., Jena, L. K., \&Kumari, I. G. (2016): Effect of work-life balance on organizational citizenship behavior: Role of organizational commitment. Global business review, 17(3), 15S-29S.

Quadri, A. A. (2019): Employees perception on effective reward system and motivating incentives to enhance performance in Irish owned restaurants: A case study 
of reward management system in Comet Restaurant Dublin (Doctoral dissertation, Dublin Business School).

Sandhya Nair, G. S. (2013): A study on the effect of quality of work life (QWL) on organizational citizenship behavior (OCB)-With Special reference to College Teachers is Thrissur District, Kerala. Integral Review: A Journal of Management, 6(1).

Shazly, M., \&Fakhry S.F. (2014): Nurses' perception of the quality of nursing work life and related priorities for improvement in Ain Shams University specialized hospital. Journal of American Science, 10(1), 123-131.

Soto, C. J., John, O. P., Gosling, S. D., \& Potter, J. (2011): Age differences in personality traits from 10 to 65: Big Five domains and facets in a large cross-sectional sample. Journal of personality and social psychology, 100(2), 330.

Suresh.D.(2013):“Quality of Nursing Work Life among nurses working in selected government and private hospitals in Thiruvananthapuram. AchuthaMenon Centre for Health Science Studies .SreeChitraTirunal Institute for Medical Sciences \& Technology .Thiruvananthapuram, Kerala.

Swaminathan, S., \&Jawahar, P. D. (2013): Job satisfaction as a predictor of organizational citizenship behavior: An empirical study. Global journal of business research, 7(1), 71-80.

Swamy, D., Swamy, N., Rashmi, S. (2015): Quality of work life, scale development and validation. The international journal of caring sciences, 8(2): 281.

Tabrsa, Gh.,Esma'eeliGivi, M., Esma'eeliGivi, H., (2010): “The factors effective on organizational commitment behavior in a military hospital". The journal of military medicine, 2(12):93-99. 


\section{سلولك المواطنة المؤسسي وجودة حياة العمل لاى الممرضين في مستثفيات بورسعيد}

أ.د/ فاطمة احمد عابد على عثمان1، أ.م.د/ سونيا محمد السيد الصباد² ، نـورا فــوزى السـيد سـليمـان

النحساس 3

أستاذ ادارة التمريض-كلية التمريض-جامعة القاهرة'، أستاذ مساعد التمريض النفسى والصحة العقلية ـ كلية

$$
\text { التمريض - جامعة بور سعيد 2بكالوريوس تمريض- جامعة بورسعيد3 } 3
$$

\section{الـخـلاصــة}

من الضروري زيادة جودة حياة العمل للممرضات لأن هذا له القرة على تحسين سلوك المواطنة المؤسسى وضمان وجود قوة عاملة تمريضية كافية.وتقوم هذه الدراسة على اكتشاف العلاقة بين سلوك المواطنة المؤسسى وجودة حياة العمل لدى الممرضين فى مستشفيات بورسعيد.وقد تم استخدام دراسة مقارنة ارتباطية وصفية لتتفيذ هذه الدراسة حيث

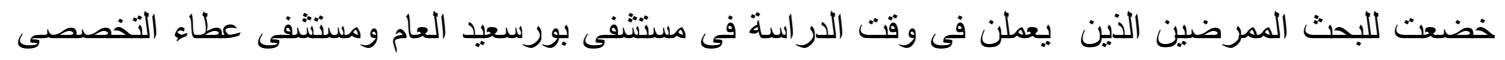

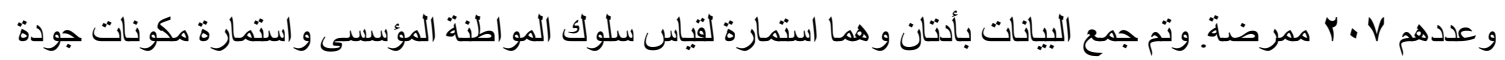
حياة العمل. وأظهرت نتائج الدر اسةأن نسبة كبيرة من الممرضات الذين تمت در استهم يتفقون على مقياس سلوك المو اطنة المؤسسى. وأن الممرضات حصلن على أعلى الدرجات من بعد الإيثار ،وعى الضمير تليها المجاملة. بينما جودة حياة

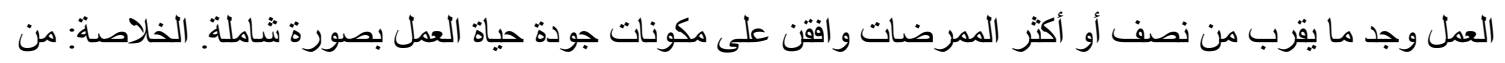
الو اضح أن نوجد علاقة ذات دلالة إحصائية بين سلوك المواطنة المؤسسى ومكونات جودة حياة العمل، وأيضا هناك

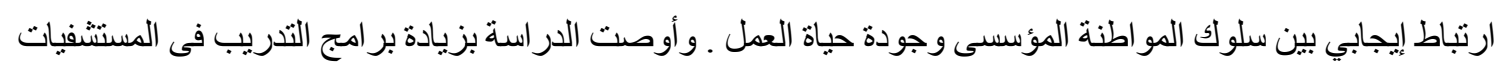
لمساعدة الممرضات فى تحقيق المهار اتجات المطلوبة وتحسين التو اصل بينهم.

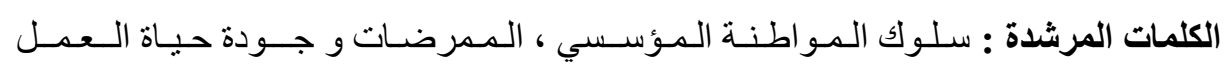

